\title{
NETWORK COARSENING DYNAMICS IN A PLASMODIAL SLIME MOULD: MODELLING AND EXPERIMENTS
}

\author{
Werner Baumgarten $^{\mathrm{a}}$, Jeff Jones $^{\mathrm{b}}$, Marcus J.B. Hauser ${ }^{\mathrm{a}}$ \\ ${ }^{a}$ Abteilung Biophysik, Institut für Experimentelle Physik \\ Otto-von-Guericke Universität Magdeburg \\ Universitätsplatz 2, 39106 Magdeburg, Germany \\ ${ }^{\mathrm{b}}$ Centre for Unconventional Computing, University of the West of England \\ Coldharbour Lane, Bristol, BS16 1QY, United Kingdom
}

(Received March 9, 2015)

The giant unicellular slime mould Physarum polycephalum forms an extended network of stands (veins) that provide for an effective intracellular transportation system, which coarsens in time. The network coarsening was investigated numerically using an agent-based model and the results were compared to experimental observations. The coarsening process of both numerical and experimental networks was characterised by analyses of the kinetics of coarsening, of distributions of geometric network parameters (as, for instance, the lengths and widths of vein segments) and of network topologies.

DOI:10.5506/APhysPolB.46.????

PACS numbers: 05.65.+b, 87.17.-b, 87.17.Aa, 89.75.Fb

\section{Introduction}

Physarum polycephalum is a well-studied, giant, multinucleated, single amoeboid cell, which has developed into a prototypical system for investigating two-dimensional transportation networks. The morphology of the plasmodium of $P$. polycephalum consists of an apical zone and an adaptive vein network [1], through which protoplasm and nutrients are continuously pumped back and forth. This peristalis-driven phenomenon is known as shuttle streaming. The adaptive vein network of $P$. polycephalum forms a regular graph (in the mathematical sense) [2,3], which is known to solve several graph theoretical problems, like finding the shortest path in a maze [4], constructing Steiner minimum trees [5], or even mimicking the topology of road and railway networks [6-10]. The biological functionality of the vein network is to provide for an effective transport of protoplasm. Recently, it

k:/June(EG-N)_2015/Baumgarten_3143/Baumgarten.tex (1001) May 22, 2015 
has been shown that the self-similar vein network is hierarchically structured with respect to its transport efficiency [11]. Such a functionality demands a continuous and well-organised optimisation of the vein network. To understand the criteria according to which these networks are optimised, numerical simulations have been performed using a variety of models [6-8, 12-22].

The proposed models of the network topology of $P$. polycephalum were developed in order to address different questions. Hence, the nature of these models varies. A first group of models has been developed to study the optimisation of the transportation capabilities (i.e., the protoplasmic flow) of the plasmodial vein networks. These models consider the networks as following Kirchhoff's rules and supporting Poiseuille flow of protoplasm. Usually, a network of a preselected topology is given and the change in the weights of the connectivities between nodes (mimicking the intensity of the flux between two nodes) is studied as some conditions are varied [6, 8, 12-15]. These simulations provide networks with altered vein strengths, however, they do neither consider the annihilation of veins nor any topology changes during the development of the network.

A second group of models has been developed to investigate the synchronisation of peristaltic pumping in a network [13, 16]. These models treat the nodes (branching points of veins) as coupled oscillators and focus on the nature of the synchronisation patterns obtained in the network. Again, modifications of the network topology are generally not addressed by such networks.

A third group of models has been proposed to study changes in the topology of the vein networks of $P$. polycephalum [17-22]. These models are either cellular automata [17], agent-based models [18, 19], or even hybrid agent-cellular automaton schemes [20-22]. Numerical studies using these models focused on the morphologies of developing networks [22], mimicking the growth and morphology of the plasmodium either under different environmental conditions [17], or in presence of multiple food sources [20-22]. These models have been used to simulate $P$. polycephalum's ability to solve mazes and to approximate Steiner minimum trees [20].

The multi-agent model introduced in Ref. [18] uses a mobile particle approach to approximate the self-assembly, formation and subsequent adaptation of $P$. polycephalum transport networks. The model was introduced to explore the potential role of spatially implemented material-based unconventional computing substrates [23-25]. The motivation for this approach was inspired by the P. polycephalum plasmodium itself, which exhibits complex behaviour emerging from only simple component parts and interactions (and, importantly, has no special or critical components). It may thus be described as a 'bottom-up' modelling approach. Although other modelling approaches, notably cellular automata, also share these motivations and 
properties, the direct mobile behaviour of the agent particles renders it more suitable to reproduce the flux within the plasmodium. The model is notable for the self-assembly of transport networks which emerge from an initially random distribution of particles. These networks were found to exploit Local Activation and Lateral Inhibition (LALI) dynamics, (where Lateral Inhibition was indirectly implemented by substrate depletion) and, subsequently, reproduced a wide range of Turing-type reaction-diffusion patterns [26]. Furthermore, these networks also exhibit physical properties such as network minimisation, the formation of Plateau angles and the observation of von Neumann's law [27].

In contrast to the first two groups of models, the cellular automata and agent-based models not only consider the formation of novel veins but also consider their subsequent morphological adaptation and annihilation. Annihilation of veins, in fact, is a hallmark of the coarsening of networks. In contrast to flow optimisation, the coarsening of the vein networks of $P$. polycephalum has, so far, received much less attention in simulation studies. When monitoring a fixed area of the network, coarsening is observed when the plasmodium propagates. It coarsens continuously until, eventually, the slime mould leaves the monitored domain altogether. During coarsening several morphological parameters of the vein network change, for instance, the density of veins, the number of nodes (i.e., branching points of veins) and the mean length of the vein segments. Therefore, one should require that any model for the coarsening dynamics reproduces three main features, namely (i) the changes in the geometry of veins, (ii) the annihilation and disappearance of nodes, and (iii), in the long run, the network should disappear completely or collapse to a single point.

In the present paper, we investigate the evolution of $P$. polycephalum vein networks and focus on the coarsening dynamics of originally dense networks. We consider the morphology of the network, the distributions of typical network parameters, and how these properties change during the coarsening of the network. We perform numerical simulations using the multi-agent based model, recently proposed by Jones [18], and compare the numerical results to findings obtained from experiments. To this purpose, we first briefly introduce the multi-agent based model [18]. Next, we present the materials and methods used in the experiments and to perform the network graph analysis. The subsequent section reports on the results on the coarsening processes in both the simulated and experimental networks, respectively. Finally, we discuss the obtained results. 


\section{Multi-agent model of the $P$. polycephalum plasmodium}

The multi-agent model of $P$. polycephalum uses a population of coupled mobile particles with very simple behaviours within a diffusive lattice [18]. The lattice stores particle positions and the concentration of a local diffusive factor referred to generically as chemoattractant.

The function of this chemoattractant is to reproduce the sol flux within the plasmodium. Particles deposit the chemoattractant factor when they move and also sense the local concentration of the factor during the sensory stage of the particle algorithm. The particles are thus indirectly coupled by the diffusive factor. This is a simple approximation of the changing composition of the $P$. polycephalum plasmodium whereby collective particle positions represent the global structure of the material (gel phase), and collective particle movement represents the flux within the plasmodium (sol phase).

In this article, the particles reside within a circular virtual 'Petri dish' inside a lattice $400 \times 400$ pixels in size. The initial population size was composed of 25000 particles, initialised at random positions and with random orientations.

\subsection{Generation of model plasmodium cohesion and morphological adaptation}

The behaviour of each particle occurs in two distinct stages, the sensory stage and the motor stage. In the sensory stage, the particles sample their local environment using three forward biased sensors whose angle from the forward position (the sensor angle parameter, SA), and distance (sensor offset, SO) may be parametrically adjusted (Fig. 1 (a)). The sampling area $A$ is thus given as

$$
A=\frac{\mathrm{SA}}{360^{\circ}}(\mathrm{SO})^{2} \pi .
$$

The offset sensors generate local indirect coupling of sensory inputs and movement to generate the cohesion of the material. The SO parameter acts as a scaling parameter and distance is measured in pixels. A minimum distance of 3 pixels is required for coupling to occur and coupling strength increases with SO. For the experiments in this article, we fixed the values of $\mathrm{SA}$ and RA to $67.5^{\circ}$ and varied the values of SO. During the sensory stage, each particle changes its orientation to rotate (via the parameter rotation angle, RA) towards the strongest local source of chemoattractant (for example, rotating to the right in Fig. 1(b)). Variations in both SA and RA parameters have been shown to generate a wide range of reaction-diffusion patterns [26] and for these experiments, we concentrate on a particular range of SA and RA parameters which have been shown to generate network assembly and adaptation [27]. 

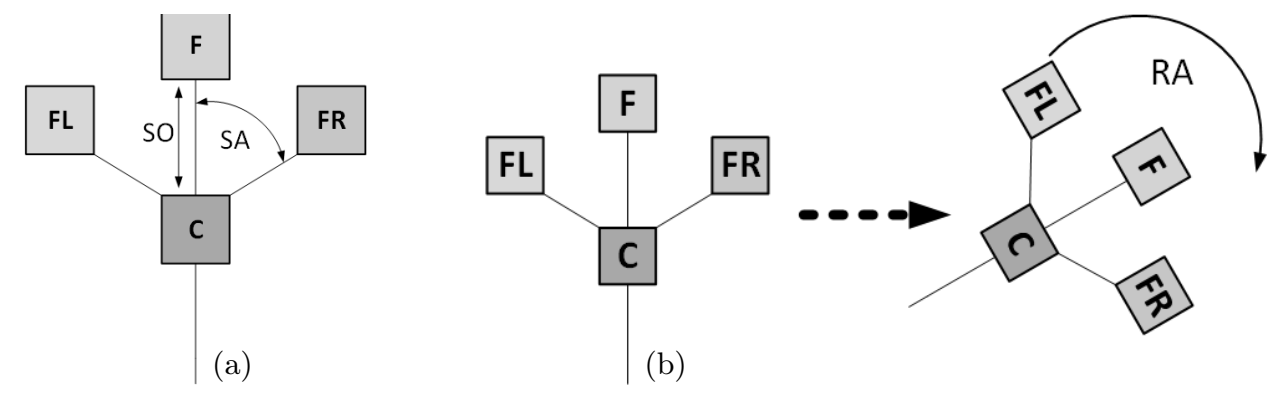

Fig. 1. Architecture of a single particle detailing the three sensory parameters. (a) Morphology showing agent position ' $\mathrm{C}$ ' and offset sensor positions (FL, F, FR, that stand for forward left, forward, and forward right, respectively) and the SO and SA parameters, (b) Effect of the RA parameter of agent orientation.

After the sensory stage, each particle executes the motor stage and attempts to move forwards in its current orientation (an angle from $0-360^{\circ}$ ) by a single pixel. Each lattice site may only store a single particle and particles deposit chemoattractant into the lattice ( 5 arbitrary units per step) only in the event of a successful forwards movement. If the next chosen site is already occupied by another particle, move is abandoned and the particle selects a new randomly chosen direction. Selection of a new direction in response to obstruction prevents the build-up of momentum within the particle population. This ensures fluid network adaptation but prevents the accumulation of different regions of flux within the population, and so the emergence of oscillatory movement is not generated. This can be achieved by removing the condition of changing direction, causing oscillatory domains to emerge and grow [28], however this is outside the scope of this article.

Diffusion of the attractant left by particle movement in the lattice was implemented at each scheduler step and at every site in the lattice in parallel via a simple mean filter of kernel size $3 \times 3$. Damping of the diffusion distance, which limits the distance of chemoattractant gradient diffusion, was achieved by multiplying the mean kernel value by 0.9 per scheduler step.

\subsection{Adaptation of model plasmodium population size}

Adaptation of the population size was implemented via tests at regular intervals. The frequency at which the growth and shrinkage of the population was executed determines the turnover rate for the population. The frequency of testing for growth was given by the $G_{\mathrm{f}}$ parameter and the frequency for testing for shrinkage is given by the $S_{\mathrm{f}}$ parameter. Both $G_{\mathrm{f}}$ and $S_{\mathrm{f}}$ were set to 5. Growth of the population was implemented as follows: If there were between $G_{\min }(0)$ and $G_{\max }(10)$ particles in a local neighbourhood 
(window size given by $G_{\mathrm{w}}$, in this case $9 \times 9$ ) of a particle, and the particle had moved forward successfully, a new particle was created if there was a space available at a randomly selected empty location in the immediate $3 \times 3$ neighbourhood surrounding the particle.

Shrinkage of the population was implemented as follows: If there were between $S_{\min }(0)$ and $S_{\max }(22)$ particles in a local neighbourhood (window size given by $S_{\mathrm{w}}$, in this case $5 \times 5$ ) of a particle the particle survived, otherwise it was deleted. Deletion of a particle left a vacant space at this location which was filled by nearby particles (due to the emergent cohesion effects), thus causing the population to shrink slightly. As the process continues, the model plasmodium continues to adapt its shape and shrink further.

The model runs within a multi-agent framework running on a Windows 7 PC system. The particles act independently and iteration of the particle population is performed randomly to avoid any artifacts from sequential ordering.

\section{Material and methods}

The dehydrated form of $P$. polycephalum strain HU195 $\times$ HU200, the sclerotia, was stored up to 24 months. Sclerotia were placed on a $1.0 \% \mathrm{w} / \mathrm{v}$ (weight per volume) plain, non-nutritive agar gel (Difco BactoAgar) in a polystyrene box (size: $18 \times 25 \times 35 \mathrm{~cm}^{3}$ ) at a constant temperature of $21^{\circ} \mathrm{C}$ in the dark. The sclerotia germinated and transformed into plasmodia which expanded over the agar matrix. During growth, oat flakes (Kölln Flocken) were used to feed the plasmodium, in order to increase the plasmodial mass.

An area of about $1 \mathrm{~cm} \times 4 \mathrm{~cm}$ of the frontal zone of the expanding plasmodium was carefully cut off, and transferred into the centre of a square polystyrene Petri dish of $12 \mathrm{~cm}$ diameter, which contained $1.0 \% \mathrm{w} / \mathrm{v}$ plain, non-nutritive agar gel (Difco BactoAgar). After several hours, a network of tubular strands (veins) developed, that coarsened as the plasmodium propagated forwards. From this network, the evolution of a region of interest was observed over time. The coarsening process was monitored with a CCD Camera (Hamamatsu C3077) at a resolution of $768 \times 576 \mathrm{px}$, where $1 \mathrm{px}=$ $0.0456 \mathrm{~mm}$ (i.e. an area of $3.5 \mathrm{~cm} \times 2.6 \mathrm{~cm}$ ). Images were acquired at a frame rate of $1 / 6 \mathrm{~Hz}$ and stored in a computer for later analysis.

The experimental and simulated networks were extracted from the stored or calculated images, respectively, and subsequently analysed according to the methods described in references $[29,30]$.

\section{Results}

A typical network coarsening, as produced by the model, is depicted in Fig. 2. After the initialisation of the model, the network is formed (Fig. $2(\mathrm{a})$ ), and subsequently it begins to coarsen (Fig. 2(b)-(d)). During 
Network Coarsening Dynamics in a Plasmodial Slime Mould: Modelling . . 1007

coarsening the number of veins is reduced, and the area of the network decreases, as indicated by the red circle which encloses the vein network. During the entire coarsening process, none of the nodes or edges remain in their position.

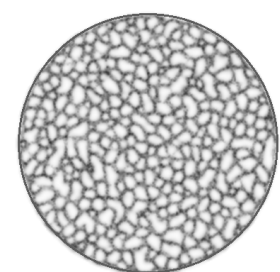

(a) $\mathbf{t}=\mathbf{5 0}$

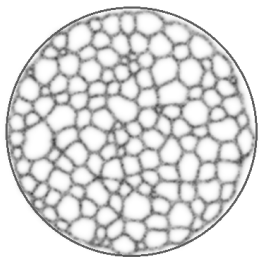

(b) $\mathbf{t}=\mathbf{3 0 0}$

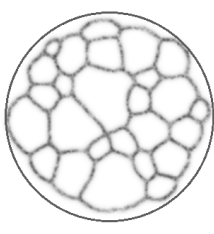

(c) $\mathrm{t}=\mathbf{1 6 5 0}$ (d) $\mathrm{t}=9075$

Fig. 2. Coarsening in the model. $\mathrm{SO}=5, \mathrm{RA}=\mathrm{SA}=67.5^{\circ}$. The red circle enclosing the network indicates the circular shape of the network. This allows the measurement of the network diameter and thus the area, where the vein network is embedded. (a) At 50 time steps (time units, t.u.), a dense network has formed. (b) At 300 t.u., the number of veins has decreased. (c) At 1650 t.u, . the vein network has almost lost its circularity. (d) At 9075 t.u., the vein network has vanished, due to the coarsening.

The coarsening of an experimentally observed vein network is shown in Fig. 3. At the beginning of the experiment, the plasmodium propagates over the agar. When it completely covers the observed area (Fig. 3 (a)), the vein network is very dense. As the plasmodium propagates, it keeps its mass (as the plasmodium migrates over a non-nutrient gel). This leads to the coarsening of the vein network (Fig. 3 (b), (c)). The coarsening is monitored until the plasmodium has moved out of the observation area.

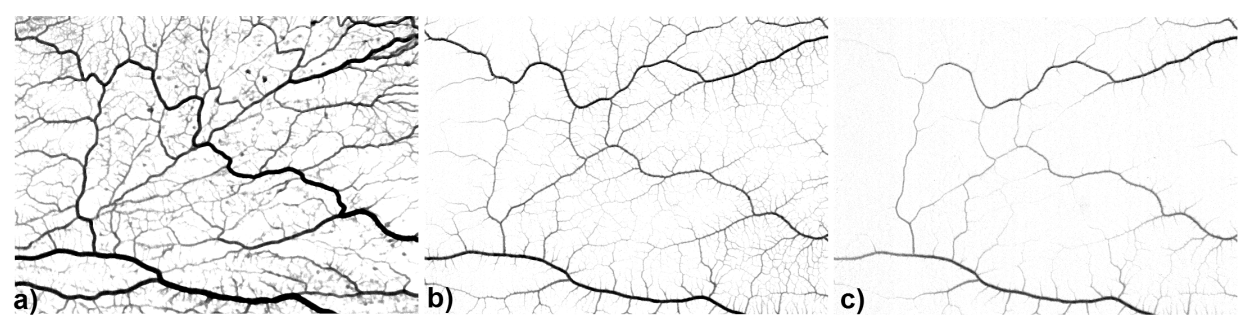

Fig. 3. Coarsening of a $P$. polycephalum vein network. The plasmodium propagates from left to right. (a) $16 \mathrm{~h}$ after beginning the experiment, the dense vein network is found in the observation area. (b) $17.5 \mathrm{~h}$. The vein network begins to coarsen. (c) $19.5 \mathrm{~h}$. Further coarsening of the vein network. After $20.0 \mathrm{~h}$ (not shown), the vein network has almost vanished. 


\subsection{Morphology of coarsening networks}

At the beginning of the simulation, the model network covers the maximum area as the particles are distributed over the entire area of interest $A_{\text {net }}$. The densely and randomly distributed particles form a dense and extended network of veins. With time, this network coarsens and the area $A_{\text {net }}$ is covered by the network shrinks. These phenomena are associated with a continuous decrease in the number of veins, a situation that is also observed in the experiments. A notable difference between the morphologies of the experimentally observed networks and the model networks is found for the widths of the strands: whether the widths of the veins are log-normally distributed in the real $P$. polycephalum networks [2], the width of the stands is uniform and invariant in the model networks.

The log-normal distribution of vein widths observed in the experiments contributes to the generation of $P$. polycephalum networks that are hierarchically and self-similarly organised with respect to their transport efficiency [11]. Hence, these networks are optimised to provide for an efficient transport of protoplasm. Similar network structures are not found in the model.

Another morphological aspect studied is the type of graph that is realised by the model and experiment. It was recently reported that the plasmodial vein networks of $P$. polycephalum form regular graphs with the unique node degree $k=3[2,3]$. In the model, by contrast, nodes of degree $k=3$ predominate, however, during the contraction of the lacunar areas delimited by the veins, nodes of higher degree (up to $k=5$ ) can also be found. Hence, model networks possess node degree distributions, and therefore they do not form regular graphs as the real networks do.

\subsection{Network area coverage}

We define the area of the smallest circle that covers the entire network as the network area $A_{\text {net }}$, and the number of all pixels belonging to veins of the network as vein area $A_{\mathrm{v}}$. The network coverage $\rho$

$$
\rho=\frac{A_{\mathrm{v}}}{A_{\text {net }}}
$$

describes the density of veins in the network area, and it is given by the ratio of the vein area to the network area. At the beginning of the simulation, the network coverage $A_{\mathrm{v}} / A_{\text {net }}$ is large, as almost $70 \%$ of the space is covered by veins or cell mass, thus yielding $\rho \approx 0.7$. With time the network coverage decreases until any branches of veins have disappeared and the shape of the last remaining vein has become circular. During this process, the area coverage converges to $\rho \approx 0.20$. Once the network consists only of a single 
circular vein, coarsening continue further and the network density $\rho$ increases again, as the circle shrinks to a point, such that $\rho \rightarrow 1$ in the long term (Fig. 4).

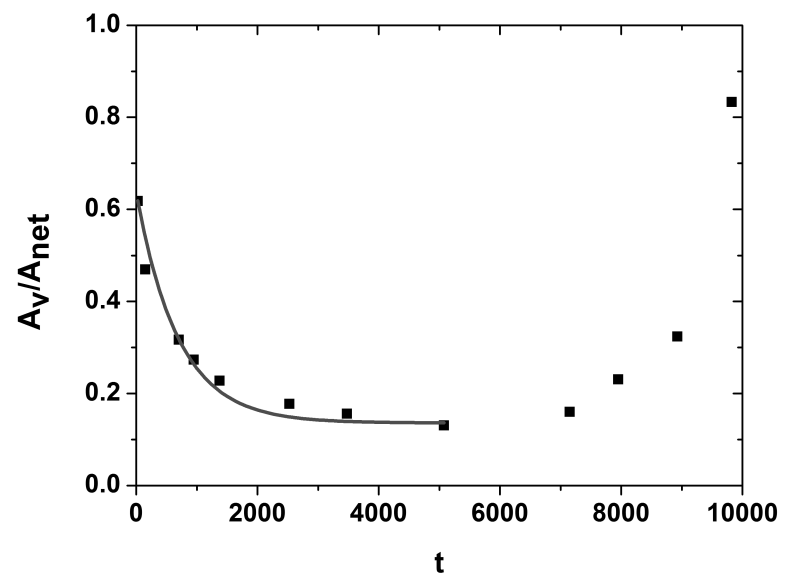

Fig. 4. Temporal evolution of the network coverage $\rho=A_{\mathrm{v}} / A_{\text {net }}$ for a model network with $\mathrm{SO}=5$ and $\mathrm{RA}=\mathrm{SA}=67.5^{\circ}$. The evolution is determined by two processes: initially, the coarsening process, where $\rho$ decays exponentially until it reaches a minimum at $t_{\mathrm{c}}=6850 \mathrm{t}$.u. (time units). Thereafter, the evolution is given by the shrinkage of the remaining circular vein to a single spot. This leads to an increase of $\rho$.

The evolution of the network coverage $\rho$ is governed by two processes, namely the coarsening of the network and, in the last stage of the coarsening, the subsequent collapse of a circular vein (Fig. $2(\mathrm{~d})$ ) to a single point. These processes are reflected in Fig. 4, where the network coverage $\rho$ at first decreases exponentially with time

$$
\rho=\rho_{0} e^{-\kappa t},
$$

until it reaches a minimum. In Eq. (3), $\kappa$ is the decay constant. The time required to reach this minimum is the coarsening time $t_{\mathrm{c}}$ that is defined as the instant where all branching points of the network have been annihilated. In Fig. 4, which was obtained using a sensor offset $\mathrm{SO}=5$, the coarsening time was $t_{\mathrm{c}}=6850$ t.u., where t.u. stands for time units (or time steps). The second process is the collapse of a circular vein to a single spot, and it occurs at $t>t_{\mathrm{c}}$. This process is associated with an increase in $\rho$.

In experiments, a similar shrinkage of the network coverage was also reported [2]. Initially, the network coverage was high, however, its value decreased as the network coarsened and finally settled to an asymptotic value of $\rho \approx 0.20$. 


\subsection{Coarsening time}

The dependence of the coarsening time $t_{\mathrm{c}}$ on the range of the sensor offset was determined from networks generated with various values of SO. Figure 5 shows that $t_{\mathrm{c}}$ shortens with increasing SO. An analysis reveals that the coarsening time $t_{\mathrm{c}}$ is proportional to $1 / \mathrm{SO}^{2}$, according to

$$
t_{\mathrm{c}}=\gamma \frac{1}{\mathrm{SO}^{2}}+t_{\mathrm{c}}(0),
$$

as demonstrated in the inset of Fig. 5. $\gamma$ is the coarsening constant, which is determined as $\gamma=140352$ t.u. $\times \mathrm{px}^{-2}$, and the offset $t_{\mathrm{c}}(0)=1422 \mathrm{t}$.u. It is worth noticing that the dimension of $\mathrm{SO}$ is that of a reciprocal diffusion constant $D$.

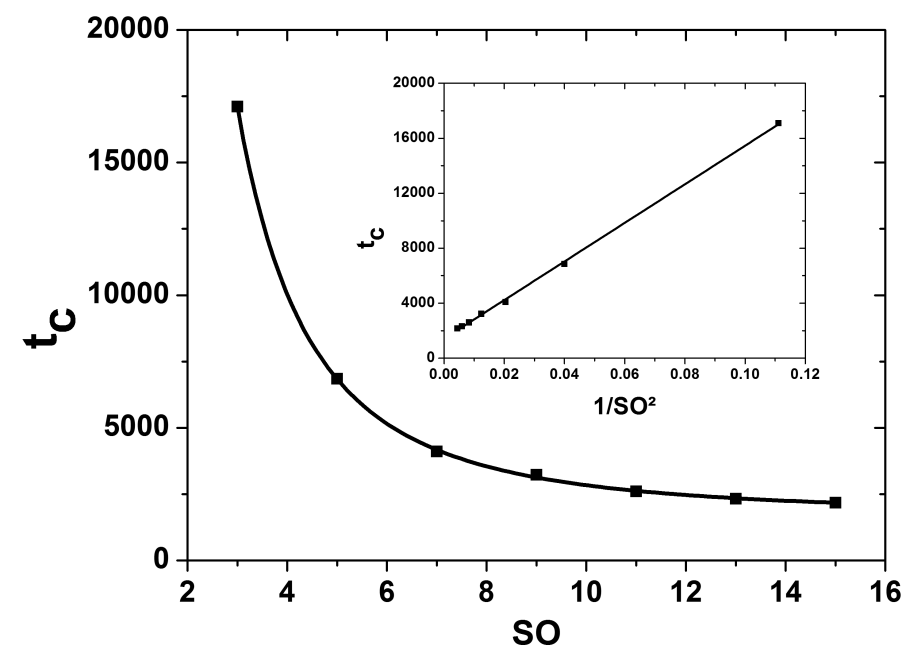

Fig. 5. Dependence of the coarsening time $t_{\mathrm{c}}$ on the sensor offset SO. A plot of $t_{\mathrm{c}}$ as a function of $\gamma / \mathrm{SO}^{2}$ is shown in the inset. This correlation is linear (see Eq. (4)) with the slope $\gamma$ as the coarsening constant obtained as $\gamma=140352$ t.u. $\times \mathrm{px}^{-2}$ and the offset $t_{\mathrm{c}}(0)=1422$ t.u. area $A$ is increased (decreased).

which states that the coarsening time decreases (increases) as the sampling 

dominant at $t / t_{\max }>0.06$. found to decrease mono-exponentially in time, as described by

$$
N=N_{0} e^{-\alpha t},
$$
ening model networks.

due to coarsening. $N_{1}+N_{2}=N_{0}$ is the number of veins at the beginning of the simulation (i.e., at time $t=0$ ). In other words, in the model, the coarsening takes place at two time scales that are characterised by the decay rate constants $\alpha_{1}$ and $\alpha_{2}$. The fast decay rate constant $\alpha_{1}$ is associated with the rearrangement of the densely distributed particles to form veins. This process is fast and leads to a drop in the network density $\rho$. Once the first veins are formed, the network coarsens at a slower rate, which is dependent on the rate constant $\alpha_{2}$. This means that in Fig. 6, the fast process (associated to $\alpha_{1}$ ) lasts until $t / t_{\max } \approx 0.06$, and the coarsening process of veins which is associated with the decay rate constant $\alpha_{2}$ becomes

The coarsening of real vein networks of $P$. polycephalum follows different kinetics than that of the model networks. The annihilation of veins was

suggesting that reduction in the number of veins follows a single process. The kinetics of this process is characterised by the decay rate constant $\alpha$. The physical process accounted by the (mono-exponential) decay constant $\alpha$ resembles that described by the (bi-exponential) decay constant $\alpha_{2}$ in coars-

The decreases in the number of veins during coarsening in both model and real vein networks are plotted in Fig. 6 . Here, a normalised number of veins $N / N_{0}$ and a normalised time $t / t_{\max }$ were used to allow for a convenient comparison of the behaviours of the model and experimental networks. 


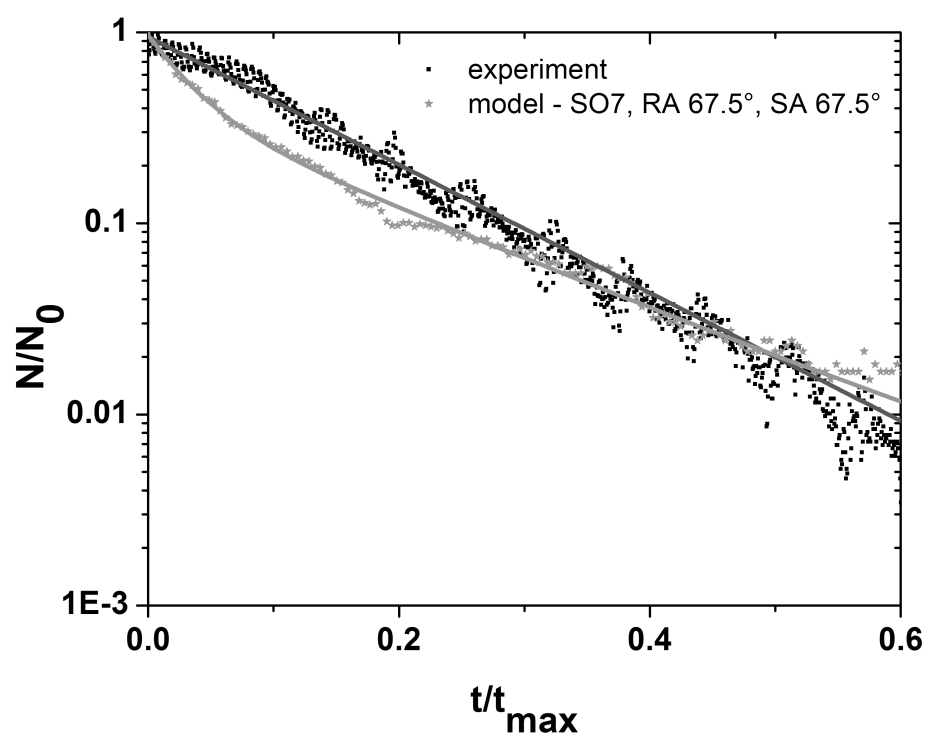

Fig. 6. Evolution of the number of veins in a coarsening network. The decay of the normalised number of veins $N / N_{0}$ in dependence of the normalised time $t / t_{\max }$. The coarsening of the model network (red stars) with $\mathrm{SO}=7$ is best fitted by a bi-exponential function (light grey/red line, with the decay constants $\alpha_{1}=-31.25$ t.u. $^{-1}$ and $\alpha_{2}=-6.25$ t.u. $^{-1}$ ). By contrast, the experimental network (black squares) presents a mono-exponential coarsening dynamics (grey/blue/line: mono-exponential fit, with $\alpha=-7.69 \mathrm{~s}^{-1}$ ).

\subsection{Evolution of the mean length of veins}

The lengths of the veins in the network are distributed log-normally, in both, the model networks (Fig. 7) and in the real networks [2]. This functional form remains constant during the entire coarsening process, only the parameter values change in time. In numerically generated networks, the log-normal function fits to the length distribution of veins to a good agreement as long as $\mathrm{SO}$ is kept small (i.e. $\mathrm{SO} \leq 7$ ). With increasing $\mathrm{SO}$ values the peak of the function becomes sharper, increasingly deviating from the typical log-normal distribution.

With time, the mean length $\langle l\rangle$ of the veins increases almost linearly in both model and real networks (Fig. 8). This can be explained by the removal of nodes from the network, which leads to both a reduction in the number of veins and an increase in their lengths. P. polycephalum continuously optimises its plasmodial vein network, resulting in the annihilation of several nodes of the vein network, such that mean vein length $\langle l\rangle$ increases. In the model, lacunar areas between the veins shrink and nodes are continuously 
removed, causing an increase in the lengths of the adjacent veins. As the network coarsens, these processes lead to a decrease of the number $N$ of veins and to an increase of the mean vein length $\langle l\rangle$.

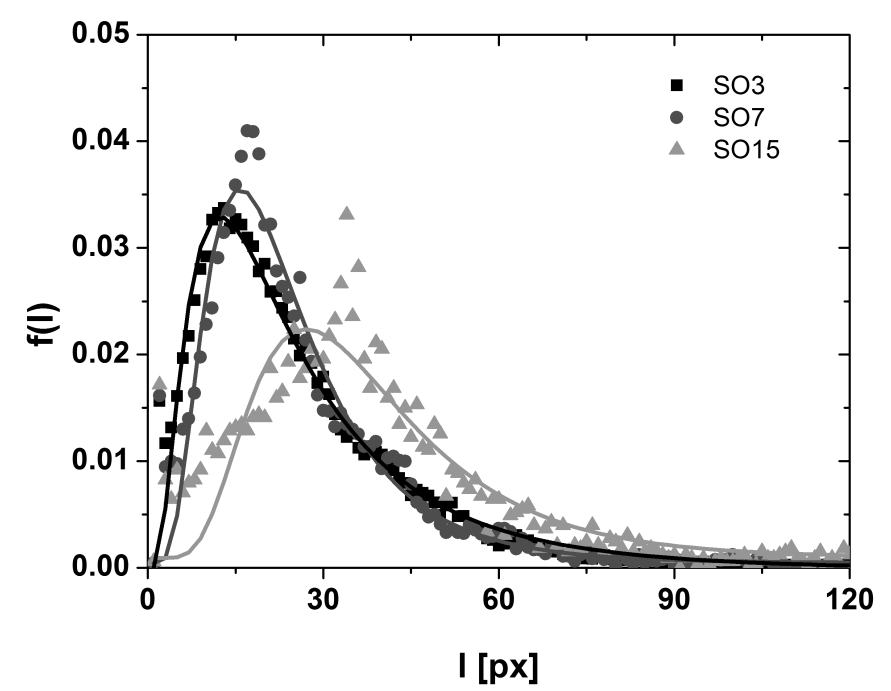

Fig. 7. Distribution of lengths of veins in simulations for $\mathrm{SO}=3$ (squares), $\mathrm{SO}=7$ (circles), and $\mathrm{SO}=15$ (triangles). The values of $\mathrm{RA}=67.5^{\circ}$ and $\mathrm{SA}=67.5^{\circ}$ were held constant. Log-normal distributions were fitted to the data (lines).

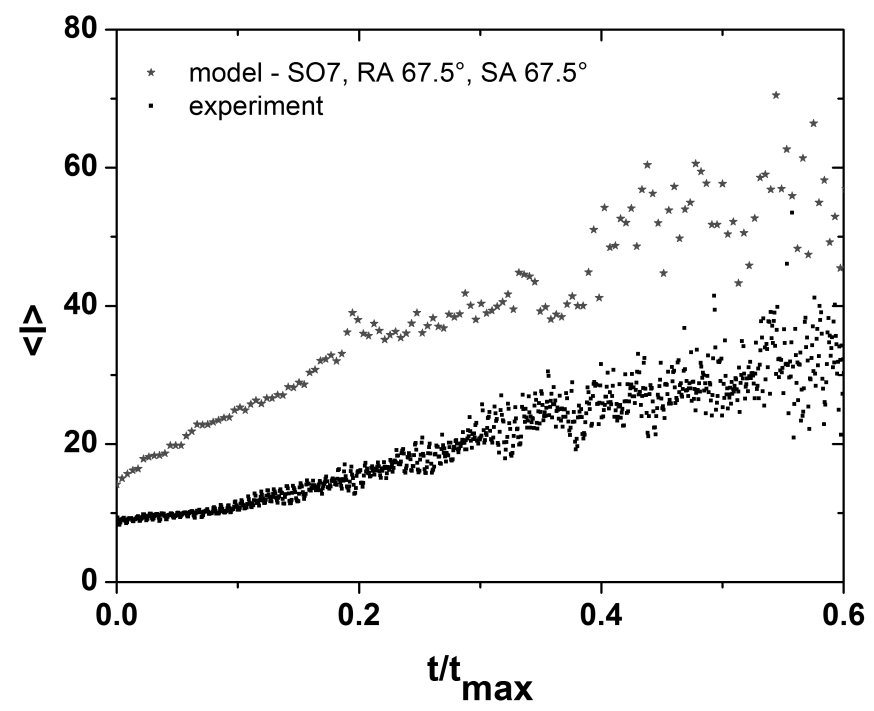

Fig. 8. Evolution of the mean vein length $\langle l\rangle$ as a function of the normalised time $t / t_{\max }$. Red stars show the data obtained from model networks, whereas black squares represent data obtained from experiments. 
The mean length $\langle l\rangle$ and the number of veins $N$ were found to be correlated through the power law

$$
\langle l\rangle=\eta N^{\beta},
$$

as revealed by Fig. 9. The exponents $\beta$ obtained from the coarsening model and experimental networks were $\beta=-0.41$ and $\beta=-0.35$, respectively, suggesting a similar, but not identical coarsening dynamics.

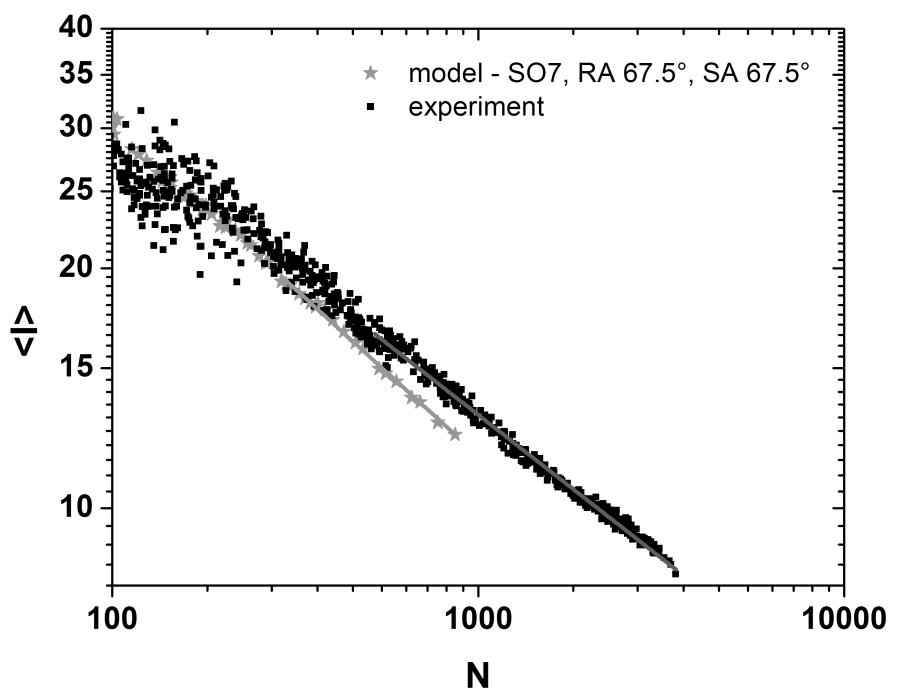

Fig. 9. Correlation between the number of veins $N$ and the mean vein length $\langle l\rangle$. Red stars and lines represent the model data and the corresponding fit of Eq. (8) to the data, respectively, whereas black squares and the light grey/blue line represent the experimental data and the corresponding fit of Eq. (8) to the data, respectively.

\subsection{Mean width of the veins}

The mean width $\langle w\rangle$ of veins remains constant in time in both the model and the experiment (Fig. 10). However, the mechanisms leading to a constant mean vein width $\langle w\rangle$ are different in the model and experimental networks. In the model, the width of veins is determined by the values of SA, RA and SO. Once these values are set, they remain fixed during the entire simulation, and so does the mean width $\langle w\rangle$ of the veins. This contrasts with the situation encountered in the experiments where the widths of the veins are distributed log-normally $[2,3]$ at all stages of the coarsening process. Interestingly, however, the mean width $\langle w\rangle$ of veins in the experiment also remains nearly constant during the coarsening process, since the log-normal distributions of the vein widths are narrow and the mean of the distribution always settles at a small value of $w$. 


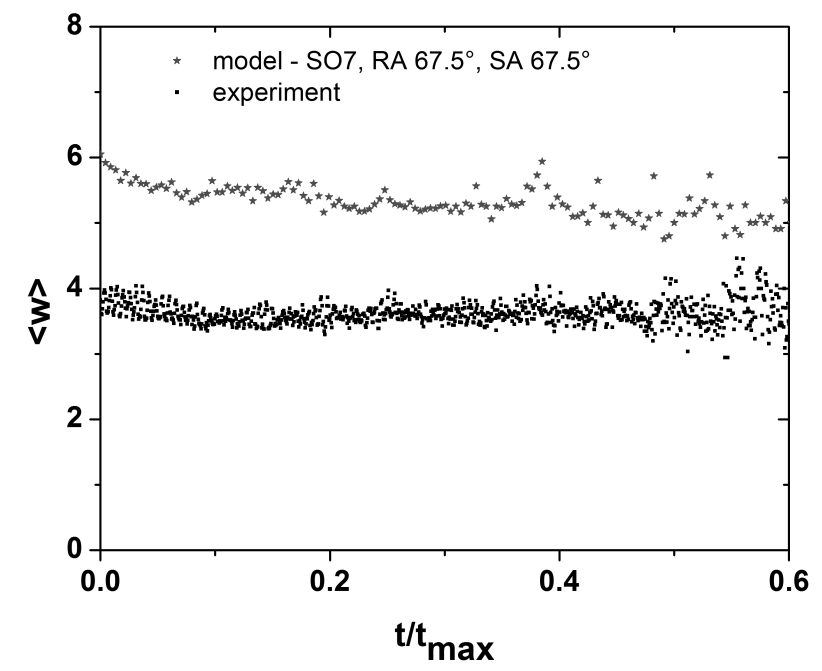

Fig. 10. Temporal evolution of the mean vein width $\langle w\rangle$ in model (red stars) and experimental (black squares) networks. Interestingly, $\langle w\rangle$ is constant in both cases. and simulated networks [22].

\section{Discussion}

Coarsening is a process that was observed in studies of $P$. polycephalum vein networks, which are optimised with respect to the transport efficiency of the protoplasm and nutrients transporting veins. Whereas the coarsening process and its dynamics have been characterised in a series of studies $[2,22,29,30]$, simulation studies of this process are scant. In fact, this has been attempted by Gunji and coworkers, who have presented a model (the vacant particle-shrinkage model) that accounts for the coarsening of an initially very dense to a sparse network that connects nutrient sources deposited on the arena (Petri dish) [22]. This setting reproduces experiments as reported in Ref. [31]. Gunji et al. have also compared the coarsening dynamics obtained in numerical simulations to that of laboratory experiments by studying the temporal evolution of some network parameters, for instance the network area, the number of loops, and area closure of the experimental

In the present article, we have performed a detailed examination of the coarsening dynamics as presented by a frequently used agent-based model for P. polycephalum network $[18,19]$. Results of the numerical studies were compared to those obtained from experiments. We found that the model reproduces a series of features seen in the coarsening of $P$. polycephalum to good agreement, while some discrepancies remain. Good agreement was achieved for the distribution of the lengths of the veins in the network, that were found to obey log-normal distributions in both, experiments and 
numerical simulations. Furthermore, a good agreement was also observed in the evolution of the mean vein length $\langle l\rangle$, which was found to correlate to the number of veins $N$ in the network by a power-law function in both, experiments and simulations. The values of the exponents $\beta$ were quite similar as well.

Another point where numerical and real networks behave similarly is the development of the network coverage $\rho$. Both experiments and simulations reveal that the network coverage decreases in time until it approaches a value of $\approx 0.2$ in both experimental and numerical networks. However, in experiments this value of $\rho \approx 0.2$ is asymptotic, whereas in the numerical simulations the area coverage $\rho$ increases again as soon as all branching points have been removed from the network, and only a single shrinking circular vein remains. This difference can be explained by different problem settings studied in the experiments at the one hand, and in the numerical studies at the other. The experiments were designed to elucidate the dynamics of a freely migrating giant plasmodium on a nutrient-free gel substrate. Ultimately, the scarce, propagating network leaves the region of observation. On the other hand, the agent-based model was originally designed to reproduce a scenario where a dense matrix of protoplasm is spread on a substrate that contains a few nutrient sources. In such a situation, the plasmodium does not migrate. In the long term, a plasmodium located in a nutrient- and stimulus-free setting (as studied in this paper) rather contracts to a single spot.

These different settings lead to some disparities in the coarsening of experimental and numerical networks. The most pronounced difference lies in the kinetics of the number of veins $N$ in the network: whereas $N$ decays mono-exponentially in the experiments, the decay of $N$ in numerical networks is bi-exponential. In the experiments, one considers the evolution of the number of veins in the network area. That is, the formation of veins in the transition zone between the apical and the network zones of the plasmodium [1] are not taken into account. By contrast, the initial condition used in numerical simulations corresponds a plasmodium that is entirely and densely covered by tiny veins, as it is the case of the transition zone. Therefore, the simulated networks account for two processes, namely the formation of the veins and their fate in a coarsening network. Following this reasoning, the kinetics observed in the experimental networks corresponds to the network decay described by $\alpha_{2}$ (Eq. (6)) in the simulated networks.

One of the factors determining the kinetics of coarsening in the simulated networks is the area of the domain $A$ that is sensed by any agent. In fact, the coarsening occurs faster as the size of the sensing domain $A$ (and hence the value of the sensor offset parameter $\mathrm{SO}$ ) increases. This suggests that the rate of network coarsening augments with the area from which any agent 
Network Coarsening Dynamics in a Plasmodial Slime Mould: Modelling . . 1017

(i.e., any position in the network) draws information about its environment. This further suggests, that an agent approaches a more efficient vein in a more directed way as the sampling range $A$ increases.

In conclusion, the present study has provided insights in the coarsening dynamics of both the plasmodial vein network of $P$. polycephalum, and networks produced by the multi-agent model proposed in Ref. [18]. Even though the modelling approach was developed for other purposes than the study of the contemplative migration of a plasmodial vein network, the network coarsening in experiments and numerical simulations show remarkable similarities. Nevertheless, the mechanistic origins leading to the remaining differences between experiments and numerical simulations constitute an interesting challenge for further studies.

J.J. was supported by the EU research project "Physarum Chip: Growing Computers from Slime Mould" (FP7 ICT Ref 316366).

\section{REFERENCES}

[1] N. Kamiya, R.D. Allen, Y. Yoshimoto, Cell Motil. Cytoskeleton 10, 107 (1988).

[2] W. Baumgarten, T. Ueda, M.J.B. Hauser, Phys. Rev. E82, 046113 (2010).

[3] M. Ito, R. Okamoto, A. Takamatsu, J. Phys. Soc. Jpn. 80, 074901 (2011).

[4] T. Nakagaki, H. Yamada, A. Tóth, Nature 407, 470 (2000).

[5] T. Nakagaki et al., Phys. Rev. Lett. 99, 068104 (2007).

[6] A. Tero et al., Science 327, 439 (2010).

[7] A. Tero, R. Kobayashi, T. Nakagaki, Physica A 363, 115 (2006).

[8] A. Tero, R. Kobayashi, T. Nakagaki, J. Theor. Biol. 244, 553 (2007).

[9] A. Adamatzky, J. Jones, Int. J. Bifurcation Chaos 20, 3065 (2010).

[10] S. Watanabe, A. Tero, A. Takamatsu, T. Nakagaki, BioSystems 105, 225 (2011).

[11] W. Baumgarten, M.J.B. Hauser, Phys. Biol. 10, 026003 (2013).

[12] A. Tero et al., Theory Biosci. 127, 89 (2008).

[13] K. Alim et al., Proc. Nat. Acad. Sci. USA 110, 13306 (2013).

[14] X. Zhang, Y. Zhang, Y. Deng, Bioinspir. Biomim. 9, 046016 (2014).

[15] X. Zhang et al., Appl. Math. Comput. 248, 18 (2014).

[16] Y. Kagawa, A. Takamatsu, Phys. Rev. E79, 046216 (2009).

[17] A. Takamatsu, E. Takaba, G. Takizawa, J. Theor. Biol. 256, 29 (2009).

[18] J. Jones, Int. J. Unconventional Comput. 6, 125 (2010).

[19] J. Jones, A. Adamatzky, Bioinspir. Biomim. 7, 016009 (2012). 
[20] Y.P. Gunji, T. Shirakawa, T. Niizato, T. Haruna, J. Theor. Biol. 253, 659 (2008).

[21] T. Niizato, T. Shirakawa, Y.-P. Gunji, BioSystems 100, 108 (2010).

[22] Y.P. Gunji et al., J. Theor. Biol. 272, 187 (2011).

[23] J. Jones, Int. J. Unconventional Comput. 7, 423 (2011).

[24] J. Jones, A. Adamatzky, Natural Computing 13, 1 (2014).

[25] J. Jones, A. Adamatzky, Bioinspir. Biomimet. 9, 036016 (2014).

[26] J. Jones, Artificial Life 16, 127 (2010).

[27] J. Jones, Natural Computing 10, 1345 (2011).

[28] S. Tsuda, J. Jones, Biosystems 103, 331 (2011).

[29] W. Baumgarten, M.J.B. Hauser, J. Comput. Interdiscip. Sci. 1, 241 (2010).

[30] W. Baumgarten, M.J.B. Hauser, J. Comput. Interdiscip. Sci. 3, 107 (2012).

[31] T. Nakagaki, R. Kobayashi, Y. Nishiura, T. Ueda, Proc. R. Soc. Lond. B271, 2305 (2004). 